\title{
Promoting Learning and Satisfaction of Children When Interacting with an Emotional Companion to Program
}

\author{
Elizabeth K. Morales-Urrutia ${ }^{1}$, José Miguel Ocaña Ch. ${ }^{2}$, \\ Diana Pérez-Marín ${ }^{3(\bowtie)}$, and Celeste Pizarro-Romero ${ }^{3}$ \\ ${ }^{1}$ Universidad Técnica de Ambato, 182020 Ambato, Ecuador \\ ${ }^{2}$ Ejército Ecuatoriano, 182020 Ambato, Ecuador \\ ${ }^{3}$ Universidad Rey Juan Carlos, 28933 Madrid, Spain \\ diana.perez@urjc.es
}

\begin{abstract}
Teaching how to program in Primary Education has attracted a great deal of attention in the last years. However, it is still unclear the approach to achieve higher learning and satisfaction levels. In this paper, the proposal is focused on the use of an emotional learning companion called Alcody. To compare whether to insert emotional elements have an equal or more significant effect on students' satisfaction and learning than personalization and execution, 137 children between 10-12 years were randomly split into four groups for three months. The higher learning and satisfaction levels are registered for the students in the group with execution, personalization and emotion management.
\end{abstract}

Keywords: Learning companion $\cdot$ Teaching programming $\cdot$ Primary

Education · Emotions

\section{Introduction}

Teaching programming to children has attracted a great deal of research interest in the last years $[1,2]$. Many approaches are being tried to develop educational environments to teach programming concepts or to allow children to create their own programs. Another area that has received a great deal of research in the last decades is the use of learning companions [3]. A learning companion can be defined as a computer agent in the educational environment that supports the student. It can try to empathize with the student so that the student feels understood (e.g. Jake and Jane [4]).

In the review of the literature, no emotional learning companion has been found to be used to teach children how to program. Our previous work was focused on the design of the educational environment to teach Primary Education children how to program called Alcody [5]. The hypothesis is that by empowering Alcody with an emotional learning companion, the satisfaction and learning levels of the students will be increased. To test the hypothesis, an experiment with 137 children between 10-12 years was carried out in a private school in Ecuador. The higher improvement and satisfaction levels were registered for the students who used Alcody with personalization, execution and emotion management. 


\section{Alcody}

Alcody is an educational environment to teach programming to Primary Education children. The name AlCODy comes from "Algorithms and CODe". Alcody is available on-line, in Spanish, at alcody.site (a demo can be accessed with the user "diana" and the password "123"). A co-design with 66 children between 10-12 years old took place to develop the first prototypes of Alcody [5].

In this study, three new factors are under investigation because of their interest in increasing the learning gains and satisfaction of the students: execution with a new button to run the program (see Fig. 1), personalization with options to change the interface (see Fig. 2 left) and emotion identification from the dialogue with the student (see Fig. 2 right with a sample recommendation message shown by Alcody as the student has told Alcody he is sad and Alcody tries to motivate him).

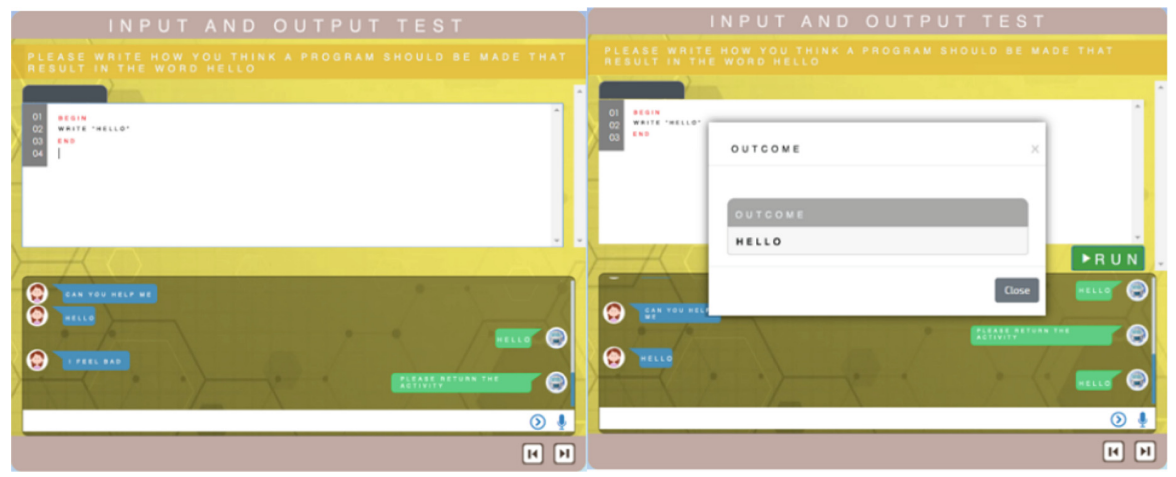

Fig. 1. Questionnaires screen without the run button (left), with the run button (right)
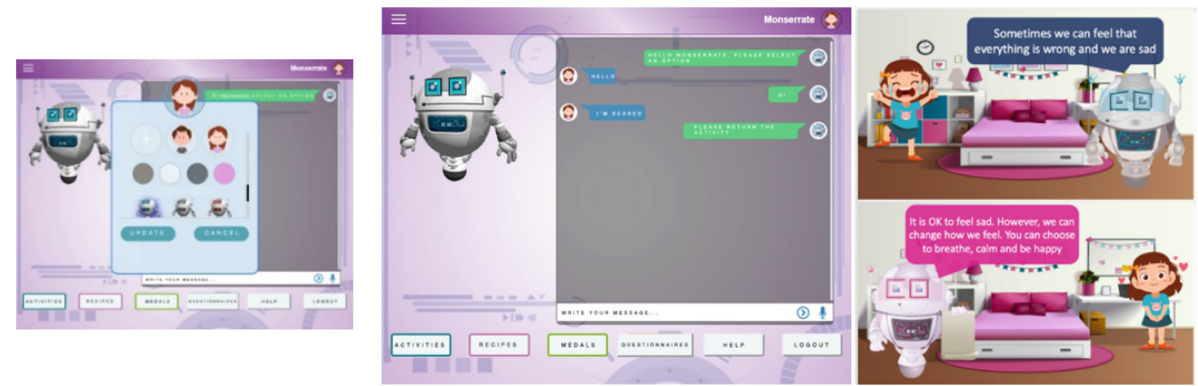

Fig. 2. Personalization (left), Emotion management (right) 


\section{Experiment}

137 children between 10-12 years enrolled in Primary Education participated in the experiment. The reason to carry out the experiment in that school was the willingness of the Head of the School to let them go to the school to teach programming to the students. Half of the sample were boys, and the other half were girls. Children were randomly split into four groups who used different options of Alcody for three months: group TEST-EPNE with execution, personalization, and without emotions; group TEST-EPE with execution, personalization and emotions; group CONTROL without personalization, execution, emotion; and, group TEST-ENPNE with execution and without personalization, emotions.

Each group was at this class with their computers (one computer per child) and they could use it (each group with their configuration of factors) for one hour per week.

The first week, all students took the same pre-test with two questions focused on the first introduction basic programming concepts to be taught: program, sequence, variable, memory, input and output. The test questions were the following:

Q1. Write a program to show "Hello" on screen. (program, sequence, output concepts)

Q2. Write a program to show the name you type on screen. (program, sequence, memory, variable, input concepts).

The last week all students took the same test with the same questions. Finally, all students were asked to fill in a satisfaction questionnaire with the following three questions: "Do you like using Alcody?", "Do you like to learn programming?" and "Would you like to keep learning how to program by using Alcody?". Two metrics have been registered: scores of the pre-post test (quantitative) and satisfaction (qualitative).

Paired samples t-test for the data are used. A comparative between pre-test and post-test is shown in Table 1. There are very significant differences for all groups $(\mathrm{p}<0.001)$. Main conclusion is that all participants improved significantly from the pre-test to the post-test. The size of this significant improvement is measured using dstatistics of Cohen. All values indicate a huge effect. To find out, whether a group

Table 1. t-test: comparative pre-post tests by groups

\begin{tabular}{l|l|l|l|l|l|l|l}
\hline Group & TEST & $\mathrm{N}$ & $\bar{X}$ & $\mathrm{sd}$ & $\mathrm{t}$ test & $\mathrm{df}$ & $\mathrm{p}$-value \\
\hline \multirow{2}{*}{ TEST-EPNE } & PRE & 32 & 0.53 & 0.40 & $\mathrm{~T}=25.142$ & 31 & $<0.001$ \\
& Post & 32 & 7.78 & 1.59 & & & \\
\hline \multirow{2}{*}{ TEST-EPE } & PRE & 36 & 0.83 & 0.89 & $\mathrm{~T}=29.250$ & 35 & $<0.001$ \\
& Post & 36 & 9.02 & 1.40 & & & \\
\hline \multirow{2}{*}{ CONTROL } & PRE & 34 & 1.62 & 0.90 & $\mathrm{~T}=11.507$ & 33 & $<0.001$ \\
& Post & 34 & 6.07 & 1.99 & & & \\
\hline \multirow{2}{*}{ TEST-ENPNE } & PRE & 35 & 0.52 & 0.59 & $\mathrm{~T}=19.432$ & 34 & $<0.001$ \\
\cline { 2 - 5 } & Post & 35 & 7.31 & 2.03 & & & \\
\hline
\end{tabular}


improved more than others, a one-way ANOVA is performed. The variation of Alcody used (this is, group variable) is statistically significant $(\mathrm{p}<0.001)$. A post-hoc analysis is used to yield more information about these differences. Tukey's HSD tests is used to compare each intervention method with every other intervention twice, exposing significant differences $(\mathrm{p}<0.001)$ between all pairs of groups except between TESTEPNE and TEST-ENPNE.

Finally, from the answers of the children to the satisfaction questionnaires, some qualitative analysis of the satisfaction levels of the groups can be performed. The groups that worked with the new factors were more satisfied using Alcody. All of them answered that they enjoyed using Alcody to learn how to program. Moreover, they showed higher desires to continue learning to program with Alcody unlike the other children who showed less interest in the platform although they could be interested in improving their programming.

\section{Conclusion}

The satisfaction and learning gains of children learning how to program can be improved by incorporating a learning companion in the educational environment. In an experiment with 137 children between 10-12 years, students using a system with execution, personalization and emotion management had the highest improvement in their scores to a pre-post programming test. Moreover, they also registered the higher satisfaction levels with respect to other group of students.

Acknowledgments. Research funded by the projects TIN 2015-66731-C2-1-R and by the Madrid Regional Government, through the project e-Madrid-CM (P2018/TCS-4307). The e-Madrid-CM project is also co-financed by the Structural Funds (FSE and FEDER).

\section{References}

1. Computer Science Teachers Association. Computer science $\mathrm{K}-8$ : building a strong foundation (2012). http://csta.acm.org/Curriculum/sub/CurrFiles/CS_K8_Building_a_Foundation.pdf

2. Jacobsen, H.: Five-years-olds learn coding in schools to prepare for future labour market. EurActiv.com - EU News \& policy debates, across languages (2014)

3. Chou, C., Chan, T., Lin, C.: Redefining the learning companion: the past, present, and future of educational agents. Comput. Educ. 40, 255-269 (2003)

4. Arroyo, I., Woolf, B., Royer, J.M., Tai, M.: Affective gendered learning companions. In: Artificial Intelligence in Education (2009)

5. Morales Urrutia, E.K., Ocaña Ch., J.M., Pérez-Marín, D., Tamayo, S.: A first proposal of pedagogic conversational agents to develop computational thinking in children. In: 5th International Conference on Technological Ecosystems for Enhancing Multiculturality (TEEM). ACM (2017) 